\title{
Automated Waste Segregation using Convolution Neural Network
}

\author{
Anagha Ravishankar ${ }^{1}$, Anvita Murthy ${ }^{1}$, Manas Sharma ${ }^{1}$, R K Chitra ${ }^{1}$, R. Anitha ${ }^{2}$ \\ Under Graduate Students ${ }^{1}$, Professor Doctor ${ }^{2}$ \\ Department of Computer Science and Engineering \\ The National Institute of Engineering, Mysuru, India
}

\begin{abstract}
:
The key to efficient waste management is to ensure segregation of waste and resource recovery. Waste is usually segregated on the basis of whether it is biodegradable or non biodegradable. A major challenge with respect to this is that waste is not segregated before collection and is thrown into dustbins nonetheless. These end up as huge piles of waste in dump yards, which needs to be segregated. Waste segregation is currently being done manually by the Municipal Corporation. These results in unsanitary working conditions for the people who need to perform this task. Despite being provided with the necessary equipment, they run the risk of catching infections from the waste they work with. Automation of this process will be beneficial for the people who work on this, by reducing health hazards.
\end{abstract}

Keywords: Object Classification, Microcontroller, Image Processing, Residual Network, Neural Network

\section{INTRODUCTION}

Machine Learning and Image Classification are technologies that have become very popular in recent times. They have a wide range of applications and are used by major fields for creating thematic maps, anomaly detection, spatial analysis etc. Machine Learning deals with systems being able to learn from past data. Image Classification refers to identifying which class an object in an image belongs to. Many Social media platforms use image classification to identify people in the images uploaded and tag them based on previous tags.

Recycling is a totally vital phenomenon in a wholesome and cleaner environment[1]. In India, 62 million heaps of rubbish is generated annually. Of this 5.6 million heaps of wastes encompass plastic substances. About 60 percentages of that is recycled each year. In addition, 11.9 million heaps are recycled from 43 million heaps of stable waste produced. Though the numbers sound good, a extreme trouble within side the recycling enterprise is the segregation of waste earlier than recycling or some other waste remedy processes [2]. In India, at present state of affairs waste isn't always segregated while gathered from households. So a variety of team of workers and attempts are made to separate this waste. In addition to this human beings working tin this atmosphere are liable to numerous infections materials because of poisonous substances generated withinside the waste. So the concept is to lower the human intervention and make this waste segregation procedure extra productive. The proposed findings are aimed to construct an image classifier that identifies the item and detects the form of waste with the use of Convolution Neural Network.

With the rise in pollution levels, segregating waste by classifying it as biodegradable and non- biodegradable is a solution to control pollution [3]. Biodegradable waste refers to waste that can be decomposed by bacteria and other living organisms. This type of waste can be recycled or used for composting purposes. Non biodegradable waste refers to waste that cannot be decomposed like plastic, and will remain stagnant to cause more harm to the environment if not treated properly. Therefore, this project aims at building a system that is able to automatically identify and classify wastes to decomposable categories. This is more efficient and faster than manually sorting the waste and classifying them, owing to the colossal amount of waste that is generated on a daily basis. The waste is transported from the bins all over the city to dump yards, where they are segregated and treated accordingly. Automating this process of segregation provides an edge over doing the same manually as it is much faster and more efficient.

\section{AUTOMATED SEGREGATION}

\section{A. PROTOTYPE}

Segregation of waste is one of the factors included in law to make it a better place for the people and the environment. Segregation of waste is important to prevent soil degradation and reduce the burden on cities. Currently in many places across the globe segregation of waste is done manually in the dump yard by the workers with lack of protective gear. This unhygienic environment leads to birth of many diseases and illness. Automating this process helps in effective disposals with very minimal human intervention.

The automated waste segregation system classifies the waste as bio degradable or non bio degradable based on image processing considering the factor of color, texture, size, shape and orientation. The waste materials are loaded onto the mechanical /conveyer belt, the camera captures the picture and sends it as an input for the deep leaning model to perform image processing, classification and detection. The microcontroller after receiving the classification output sends appropriate signals to the gate / gear to open up the path based on degradable material type. The heart of the model is the image classifier which controls and makes all the decisions. The model Resnet 34 (Residual Network) involves large number of neural layers helping in incremental learning [4].

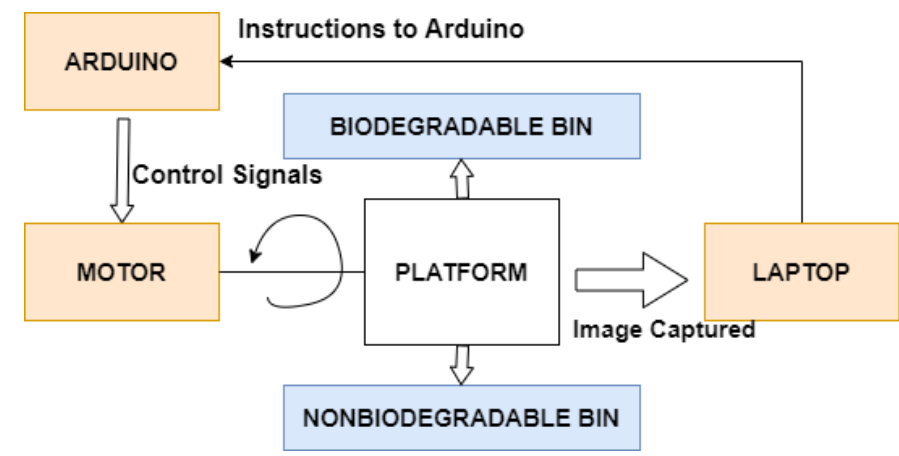

Figure.1.Automated segregation system architecture 


\section{B. HARDWARE SETUP}

The image of the waste material to be classified is captured by the web cam, transformed to $128 \times 128$ pixels and fed into model for processing. Convolution Neural Network (CNN) model is used in analyzing the captured image to produce corresponding result [5]. CNN tremendously minimizes the preprocessing attempt for an image dataset. It has the capacity to study high-degree abstractions from a raw input data. The signal triggered by the CNN model is fed to the arduino, to rotate the gears accordingly to the corresponding bio degradable or non biodegradable pits. The motor drops the waste material to the predicted bin and returns back to the original position to process the next material as indicated in Figure 3.

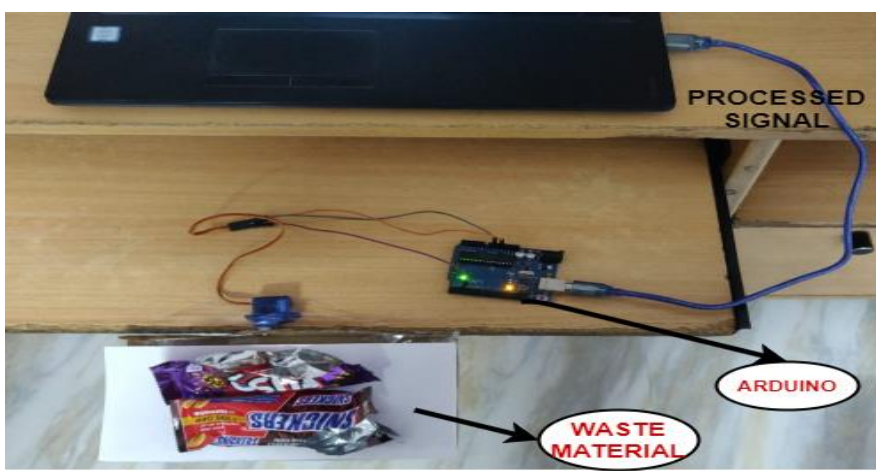

Figure.2. Waste Classification Setup

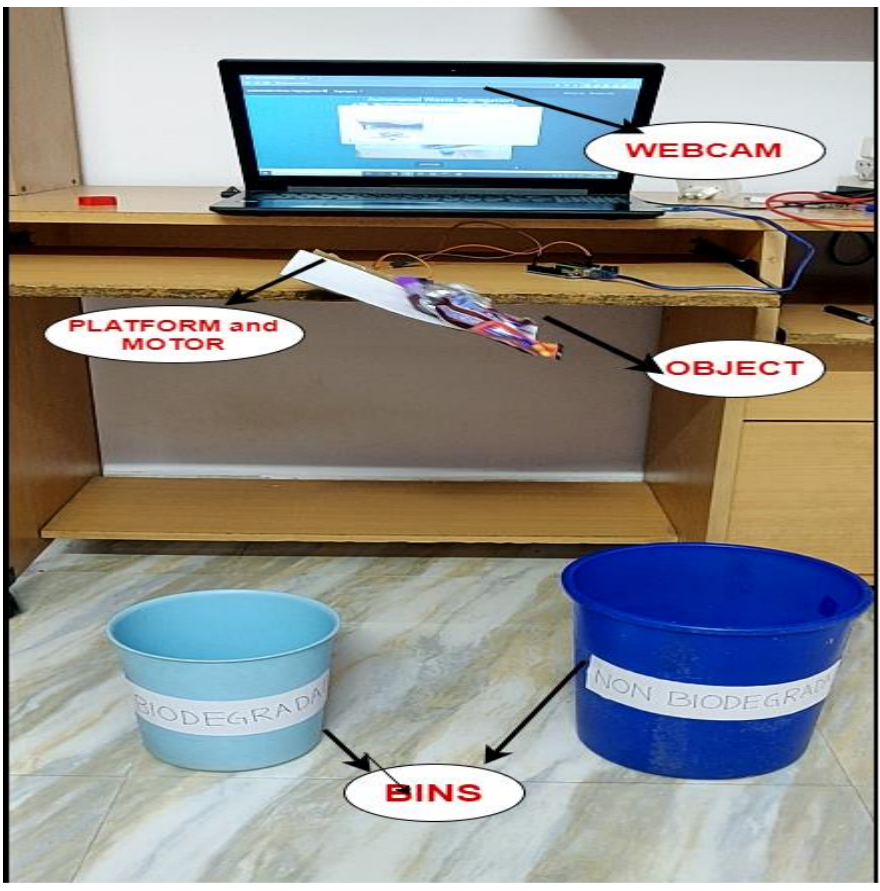

Figure.3. Platform movement after classification

\section{DATASETS AND DATA-COLLECTION}

The images used withinside the proposed project are collected from image scrapping the internet and kaggle sources. Large dataset consisting of 2566 images are used to train and test the network. The custom CNN structure calls for a huge dataset and additionally takes a number of times to train. Pre-educated models conquer those troubles as its weights are already optimized while training on the open source ImageNet dataset $[6,7]$. Though the models can't acquire $100 \%$ accuracy, its miles constructed to acquire the quality viable classifier and to decrease the mistakes to the most viable extent.
Before these datasets are put into training, the images are transformed and flipped in all directions to help the model analyze all the angles of the image. Initially an aspect ratio of $64 \times 64$ pixels were considered which yielded poorer results due to decreased pixel density, hence doubling the ratio to 128 x 128 pixels provided much better consistency and accuracy.

\begin{tabular}{|l|c|}
\hline \multicolumn{1}{|c|}{ Class } & Dataset count \\
\hline Plastic Items & 589 \\
\hline Kitchen waste, Wood and Leaves & 210 \\
\hline Pen, Paper and Glass & 649 \\
\hline Bags and Cardboards & 461 \\
\hline Wrappers, Cans and Thermocol & 385 \\
\hline Masks, Straws and Teabags & 272 \\
\hline Total Training Images & $\mathbf{2 5 6 6}$ \\
\hline
\end{tabular}

Table.1. Details of Dataset

\section{NEURAL NETWORK MODELS}

\section{A. MULICATEGORY CLASSIFICATION}

On capturing a colored image, we will be dealing with $128 \times 128 \times 3$ values because of the RGB (Red, Green, Blue) color scheme. The input layer of the neural network cannot handle multi dimensional matrices hence we pass these as values instead [12]. The neural network processes the image through several hidden layers, with the last layer having the number of neurons equal to that of the number of output classes. The convolution layer tries to extract the features and patters of the images with their filters [8]. The training sets are fit into 16 batch sizes. The multi-category model detects and classifies images into 15 categories (namely Kitchen residues, Plastic Items, Wooden Objects, Leaves, Pen, Paper, Glass Items, Bags, Cardboards, Wrappers, Cans, Thermocol, Masks, Straws and Teabags). A mapping sheet is created in python to map the classified categories of object into bio degradable and non biodegradable. This produces an accuracy of $93.20 \%$.

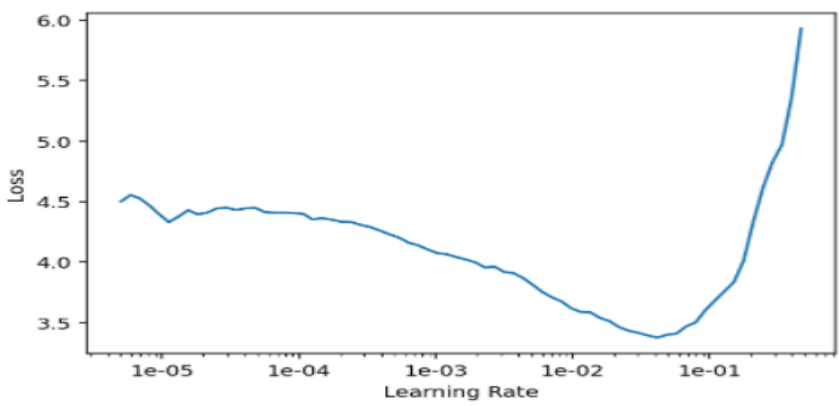

Figure.3. Loss vs. learning rate for multi-category classifier

\section{B. BINARY CLASSIFIER}

A binary classifies works in the same way as that of multicategory classifier except that the last neural network layer has only two neurons. It detects and classifies images into 2 categories only (bio degradable and non biodegradable).

Even though this model produced an accuracy of $95.09 \%$, it is difficult to debug and handle misclassified objects. With this intention in mind proceeding with the multi-category model would be a better choice. 


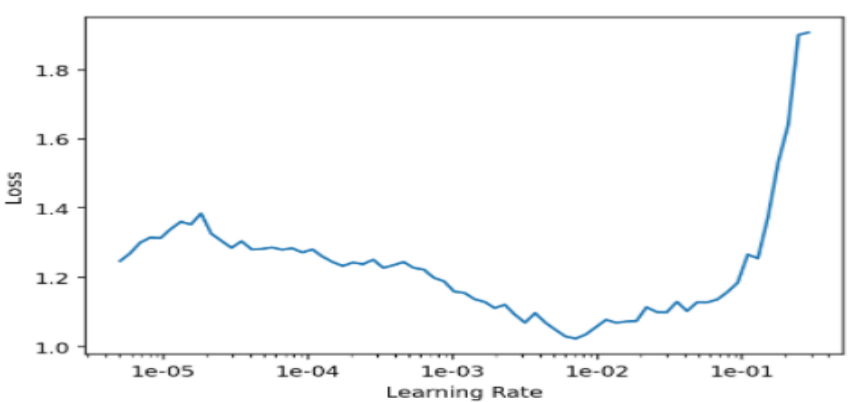

Figure.4. Loss vs. learning rate for Binary classifier

\section{RESULTS AND DISCUSSIONS}

The two key concerns for the classifier was the small size of the dataset creating insufficient training atmosphere and an overfitting model due to much less data [9]. Image Scraping and debugging of misclassified snap shots are done to eradicate these problems. On adopting the multi-category classifier, the subsequent step is to evaluate the overall performance of the advanced version the usage of a few assessment metrics. Accuracy is the most normally used and satisfactory output metric to evaluate numerous models. It is an easy ratio of successfully predicted samples to overall range of samples.

$$
\text { Accuracy }=\frac{\mathbf{T P}+\mathbf{T N}}{\mathbf{T P}+\mathbf{T N}+\mathbf{F P}+\mathbf{F N}}
$$

Where TP $=$ True Positives, $\mathrm{TN}=$ True Negatives, $\mathrm{FP}=$ False Positives, FN = False Negatives. True positive and True negatives are the correctly predicted outputs whereas False Positives and False Negatives are incorrectly predicted values. We want to decrease False Positives and False Negatives to construct a higher accuracy model. A confusion matrix is a table frequently used to outline a category model's output on a group of check statistics for which the real values are known. It permits the output of a set of rules to be visualized as depicted in Figure 5.

The final output of the multi-category classifier after mapping and capping are two outputs, one as biodegradable and the other as non biodegradable. After successful training, this model can classify different types of dry waste real time images available in the dumpster before segregation.

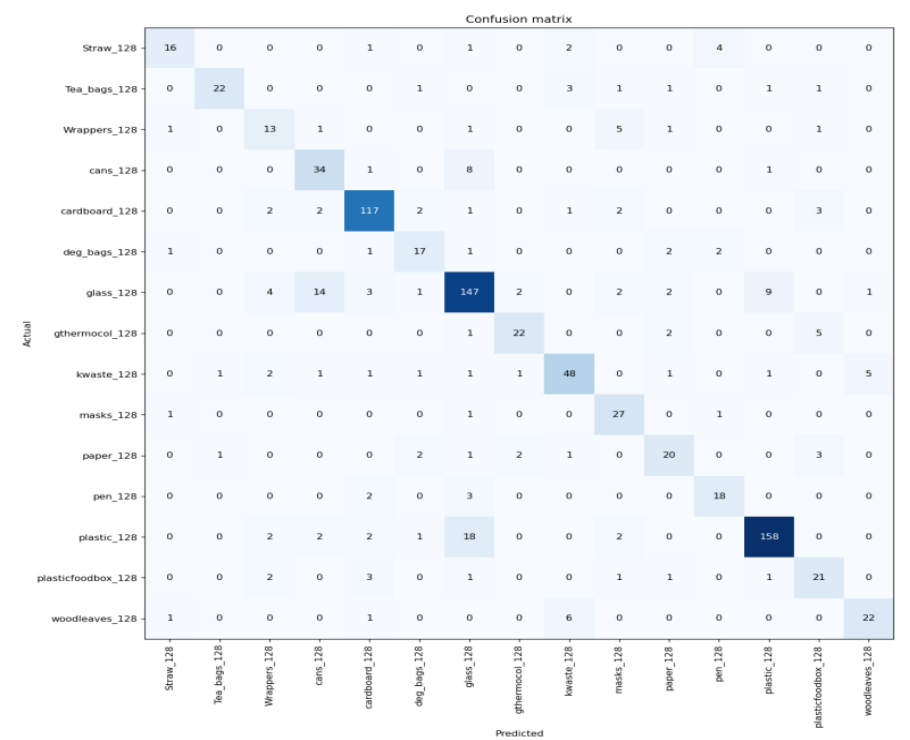

Figure.5. Confusion Matrix for multi-category classifier

\section{CONCLUSION}

In this research, our main goal is to segregate the waste materials using machine learning. The process of waste segregation after collection is crucial in controlling pollution. Making this process manual could be time consuming and extremely cumbersome. Automating this process can help efficiently segregate waste using machine learning methods like object detection to detect waste material and object classification to determine if the waste material is biodegradable or non biodegradable. This helps in effectively reducing and recycling waste materials creating a healthy environment. This prototype can be brought into mass level with permission from Municipal Corporation. This can be extended to further segregate non biodegradable waste into recyclable and non recyclable. Effectively implementing this system brings the slogan "GO GREEN, There is no planet B" to life.

\section{REFERENCES}

[1] He K, Zhang X, Ren S and Sun J, 2016. "Deep Residual Learning for Image Recognition", IEEE Conference on Computer Vision and Pattern Recognition (CVPR), Las Vegas, NV, pp. 770-778.

[2] Chandaluru Priyanka, Sri Ramya P. 2020. Image Based Classification of Waste Material Using Convolution Neural Network, International Journal of Advanced Science and Technology Vol. 29, 5, pp. 2967 - 2975

[3] Anjali Pradipbhai Anadkat, Monisha B V, Manasa Puthineedi, Ankit Kumar Patnaik, Shekhar R and Riyaz Syed. 2019. "Drone based Solid Waste Detection using Deep Learning \& Image Processing".

[4] Bakator M, Radosav D. 2018. "Deep Learning and Medical Diagnosis: A Review of Literature", Multimodal Technologies and Interaction.Vol. 2, 47, pp. 1-12.

[5] Yesha Desai, Asmita Dalvi, Pruthviraj Jadhav, Abhilasha Baphna.2018. "Waste Segregation Using Machine Learning", International Journal for Research in Applied Science and Engineering Technology (IJRASET)

[6] Alex Krizhevsky,Ilya Sutskever and Geoffrey E Hinton, 2010. "ImageNet Classification with Deep Convolutional Neural Networks", ImageNet Large Scale Visual Recognition Challenge.

[7] Thung, Gary and Ming Xiang Yang. 2016. "Classification of Trash for Recyclability Status.”, Corpus Id-27517432.

[8] Gao Huang, Zhuang Liu, Laurens van der Maaten and Kilian Q Weinberger. 2017. "Densely Connected Convolutional Networks", IEEE Conference on Computer Vision and Pattern Recognition. pp. 4700-4708.

[9] Karen Simonyan and Andrew Zisserman. 2015."Very Deep Convolutional Networks for Large- Scale Image Recognition", International Conference on Learning Representations (ICLR). pp. 1-14. 